\title{
Novel measure for the calibration of laser Doppler flowmetry devices
}

\author{
Andrey V. Dunaev, Evgeny A. Zherebtsov, Dmitrii A. \\ Rogatkin, Neil A. Stewart, Sergei G. Sokolovski, et al.
}

Andrey V. Dunaev, Evgeny A. Zherebtsov, Dmitrii A. Rogatkin, Neil A. Stewart, Sergei G. Sokolovski, Edik U. Rafailov, "Novel measure for the calibration of laser Doppler flowmetry devices," Proc. SPIE 8936, Design and Quality for Biomedical Technologies VII, 89360D (4 March 2014); doi: $10.1117 / 12.2035651$

SPIE. Event: SPIE BiOS, 2014, San Francisco, California, United States 


\title{
Novel measure for the calibration of laser Doppler flowmetry devices
}

\author{
Andrey V. Dunaev*a,b, Evgeny A. Zherebtsov ${ }^{\mathrm{b}}$, Dmitrii A. Rogatkin ${ }^{\mathrm{c}}$, Neil A. Stewart ${ }^{\mathrm{a}, \mathrm{d}}$, \\ Sergei G. Sokolovski ${ }^{a}$, Edik U. Rafailov ${ }^{a}$ \\ ${ }^{a}$ Photonics and Nanoscience Group, Division of Physics, School of Engineering, Physics and \\ Mathematics, University of Dundee, Dundee DD1 4HN, UK; ${ }^{b}$ State University - Education-Science- \\ Production Complex, Scientific-Educational Center of Biomedical Engineering, Oryol 302020, \\ Russia; "Moscow Regional Research and Clinical Institute "MONIKI", Laboratory of Medical \& \\ Physics Research, Moscow 12911, Russia; ${ }^{\mathrm{d}}$ Department of Imaging and Technology, Ninewells \\ Hospital, University of Dundee, Dundee DD1 9SY, UK
}

\begin{abstract}
The metrological basis for optical non-invasive diagnostic devices is an unresolved issue. A major challenge for laser Doppler flowmetry (LDF) is the need to compare the outputs from individual devices and various manufacturers to identify variations useful in clinical diagnostics. The most common methods for instrument calibration are simulants or phantoms composed of colloids of light-scattering particles which simulate the motion of red blood cells based on Brownian motion. However, such systems have limited accuracy or stability and cannot calibrate for the known rhythmic components of perfusion $(0.0095-1.6 \mathrm{~Hz})$.

To solve this problem, we propose the design of a novel technique based on the simulation of moving particles using an electromechanical transducer, in which a precision piezoelectric actuator is used (e.g., P-602.8SL with maximum movement less than $1 \mathrm{~mm}$ ). In this system, Doppler shift is generated in the layered structure of different solid materials with different optical light diffusing properties. This comprises a fixed, light transparent upper plane-parallel plate and an oscillating fluoroplastic (PTFE) disk. Preliminary studies on this experimental setup using the LDF-channel of a "LAKK-M" system demonstrated the detection of the linear portion $(0-10 \mathrm{~Hz}$ with a maximum signal corresponding to Doppler shift of about $20 \mathrm{kHz}$ ) of the LDF-signal from the oscillating frequency of the moving layer. The results suggest the possibility of applying this technique for the calibration of LDF devices.
\end{abstract}

Keywords: laser Doppler flowmetry, metrological support, calibration, measure, biotissue phantom

\section{INTRODUCTION}

Metrological calibration of optical non-invasive diagnostic devices has not been sufficiently addressed ${ }^{1}$. Laser Doppler flowmetry (LDF) is widely used to measure the "index of blood microcirculation $\left(I_{m}\right)$ " (or perfusion) in patients. These are conventionally reported in arbitrary perfusion units (PU), by detecting Doppler frequency shifts $(20-24000 \mathrm{~Hz})$ arising from the reflection of radiation from red blood cells (RBC) moving at speeds of $0.1-5 \mathrm{~mm} / \mathrm{s}$ in small vessels ${ }^{2,3}$. A major opportunity for this technology lies in the potential to utilise the data from individual devices from one manufacturer - as well as various manufacturers - to detect currently undetected blood flow defects and variances relevant for clinical diagnostics. LDF technology therefore requires standardization and metrological certification. LDF devices could be tested for accuracy and quantitative diagnostic information traceable back to international standards. Usually, in measuring techniques, these problems can be solved through the creation and standardization of special devices such as optical phantoms ${ }^{4}$ or working standards (measures, test objects) ${ }^{5}$.

\footnotetext{
*a.v.dunaev@dundee.ac.uk; phone 441382 386571; dundee.ac.uk/elecengphysics/research/photonics/photonicsnanoscience
}

Design and Quality for Biomedical Technologies VII, edited by Ramesh Raghavachari, Rongguang Liang, Proc. of SPIE Vol. 8936, 89360D · (C) 2014 SPIE · CCC code: 1605-7422/14/\$18 · doi: 10.1117/12.2035651 
Currently, the most commonly used instrument calibration techniques use phantoms (for example, "Motility standard" ("Perimed AB", Sweden)) comprising colloids of light-scattering particles which simulate the motion of RBC based on the Brownian motion of particles in solution ${ }^{6-8}$. However, such systems have low accuracy and stability, designed to reproduce only one level of perfusion, exhibiting high sensitivity to vibration artefacts and offer limited validity for use simply as relative RBC motion sensors. Current devices cannot check the operational registration of various rhythmic components of blood flow perfusion $(0.0095-1.6 \mathrm{~Hz})$. The registration accuracy of these rhythms is an important aspect of the LDF method reliability in medical practice ${ }^{9,10}$. The analysis showed that the development of the LDF method is constrained by its underdeveloped metrological basis.

The aim of this work was to develop a new principle of calibration measurement, devoid of the presented disadvantages and to study the experimental setup based on it.

\section{MEASUREMENT PRINCIPLES FOR CALIBRATION OF LASER DOPPLER FLOWMETRY DEVICES}

Today, the most common differential scheme for constructing LDF devices contains one probing and two receiving optical fibers, the difference in signal from which is used for further processing. A generalized formula for calculating perfusion $\left(I_{m}\right)$ in these LDF devices is written as an expression ${ }^{11}$ :

$$
I_{m}=k_{c} \frac{\int_{f_{\min }}^{f_{\max }} S_{i_{a c}}(f)|f| \mathrm{d} f}{i_{d c}^{2}},
$$

where $S_{i a c}(f)$ - the power spectral density function of the variable component of the photocurrent signal $i_{a c}(t), i_{d c}(t)-\mathrm{a}$ constant component of the photocurrent, $k_{c}$ - instrument (calibration) coefficient.

From this formula it follows that to obtain accurate, quantitative and comparable data from different devices, the LDF method requires standardization, metrological certification and checking of individual LDF devices for correct selection of instrument calibration coefficients $\left(k_{c}\right)$. For these purposes, except the most common approach, using colloidal solutions with light-scattering Brownian particles, there is also another research approach for metrological support of LDF devices. This approach uses alternating layers of different moving and solid materials with different known optical properties, providing a value of the light scattering, close to the values of the scattering in living biological tissues ${ }^{12-14}$. However, disadvantages of this approach include the following:

- the need to create a mechanical system motion from the drive motor layers, which reduces the potential accuracy due to inherent design limitations such as backlash and unwanted vibration;

- the inability to quickly and easily change the speed and range of oscillation amplitude of the motion layers to simulate the changing values of perfusion $I_{m}(t)$ according to known physiological events (reproducible complex modulated signal to simulate frequency rhythms of microcirculation);

- potential risk of abrasion over time of surfaces mechanically moving and rubbing of layers against each other changing optical properties leading to unstable values of perfusion $I_{m}(t)$ over time.

Analysis of existing approaches showed prospects for the reproduction of LDF signals by means of a moving diffusely scattering surface. Thus, it is possible to develop a reusable measure for calibration of LDF devices devoid of the drawbacks of the optical phantoms (test objects) based on light scattering on a solid surface using an electromechanical transducer (e.g. piezoelectric actuator) and managed by electrical signals (Fig. 1a).

We propose the design of a novel measure for the calibration of LDF devices, based on the principle of simulation of moving particles, in which an electromechanical transducer 3 converts electrical signal into mechanical vibrations in the moving diffuse reflector 2 . In order to create a constant component of the signal, laser radiation from the probe fiber (aperture of source $S_{i n}$ ) passes first through a fixed light transparent plane-parallel plate 1 .

Basic principles of the proposed novel measure for calibration of LDF devices:

- laser radiation is scattered by the oscillating diffusing Lambertian surface and receives the component of the Doppler shift;

- when probing a fixed light transparent plane-parallel plate by laser radiation, reflected back radiation does not undergo Doppler shift;

- the physical properties of the materials used and the geometry of the problem being solved allow for the 
quantification of the power of both components as well as the frequency of the reproduced Doppler components undergoing Doppler shift;

- the slope angle $\beta$ (Fig. 1b) of the optical fiber probe to allow the formation of a specific quantitative difference between the input channels and registration signals of LDF devices.
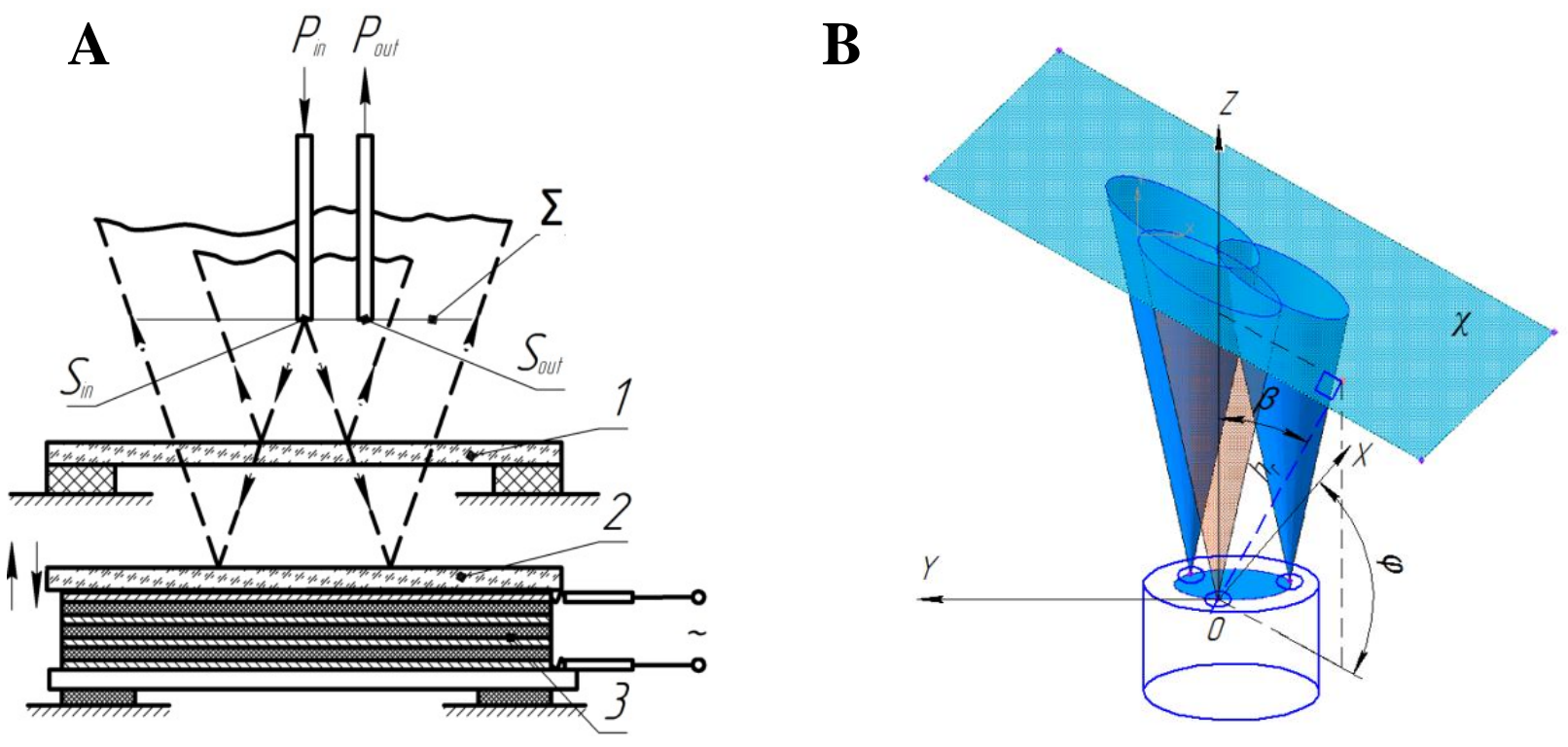

Figure 1. Principles of operation for calibration of LDF devices (a): 1 - light transparent plane-parallel plate, 2 - moving diffuse reflector, 3 - electromechanical transducer (batch piezoelectric actuator), $S_{\text {in }}$ - aperture of source, $S_{\text {out }}$ - aperture of the receiver, $\Sigma$ - plane coincidence of aperture of receiver and source; and sectional view of the probe and receivers cones by the light scattering surfaces (b): $h_{r}$ - distance from the core center of the probe fiber to the light scattering surface, $\beta$ angle of fiber-optic probe relative to the normal, carried to the plane of the light transparent plane-parallel plate, $\varphi-$ angle between the projection of the height $h_{r}$ on the plane of fiber-optic probe end and the x-axis (angle of rotation of the optical probe), $\chi$ - plane of the diffusely scattering surface.

\section{EXPERIMENTAL RESULTS AND DISCUSSION}

Pictures of the experimental setups are shown in Fig. 2. The technique uses a precision batch piezoelectric actuator P602.8SL ("Physik Instrumente GmbH \& Co", Germany) with maximum movement of less than $1 \mathrm{~mm}$. In the proposed experimental setups Doppler shift is generated in the layered structure of various solid materials with different light diffusing properties. This comprises a fixed upper layer either in the form (Fig. 2a) of a mounted continuously variable neutral density filter NDC-100C-4M ("Thorlabs", USA) or in the form (Fig. 2b) of thin polymer film ("Rosco", USA), plus a fluoroplastic PTFE disk ("Ocean Optics", USA) moved by a piezoelectric actuator. In this study, we used the LDF-channel (with $\lambda=1064 \mathrm{~nm}$ ) of the multifunctional laser non-invasive diagnostic system "LAKK-M" (SPE "LAZMA", Russia) ${ }^{15,16}$ for calibration. A typical example of a reproducible constant level of perfusion using the proposed measure is shown on the Fig. 3.

Basic findings from the theoretical and experimental studies of the proposed setup for calibration of LDF devices:

- reproducible perfusion directly proportional to the amplitude and frequency of the oscillation light scattering surface of moving diffuse reflector was achieved (Fig. 4):

$$
I_{m}=k_{p} A_{o} f_{o s c}
$$

where $A_{0}$ and $f_{\text {osc }}$ - amplitude and frequency of oscillation of the moving diffuse reflector respectively, $k_{p}-$ proportionality coefficient;

- coefficient $k_{p}$ does not depend on modes of oscillation - amplitude $\left(A_{0}\right)$ and frequency $\left(f_{\text {osc }}\right)$, and may be calculated based on knowledge of specific design parameters; 
- slope angle of the fiber probe has a range of acceptable values $0<\beta<\beta_{\max }$ (Fig. 1b), such that when exceeded causes that the reflected radiation not to be used by the receiving fiber;

- reproducible level dependence on the angle $\beta$ of the perfusion $\left(I_{m}\right)$ has a local maximum in the range of valid values $0<\beta<\beta_{\max }$ that can be calculated for specific values of the design parameters.
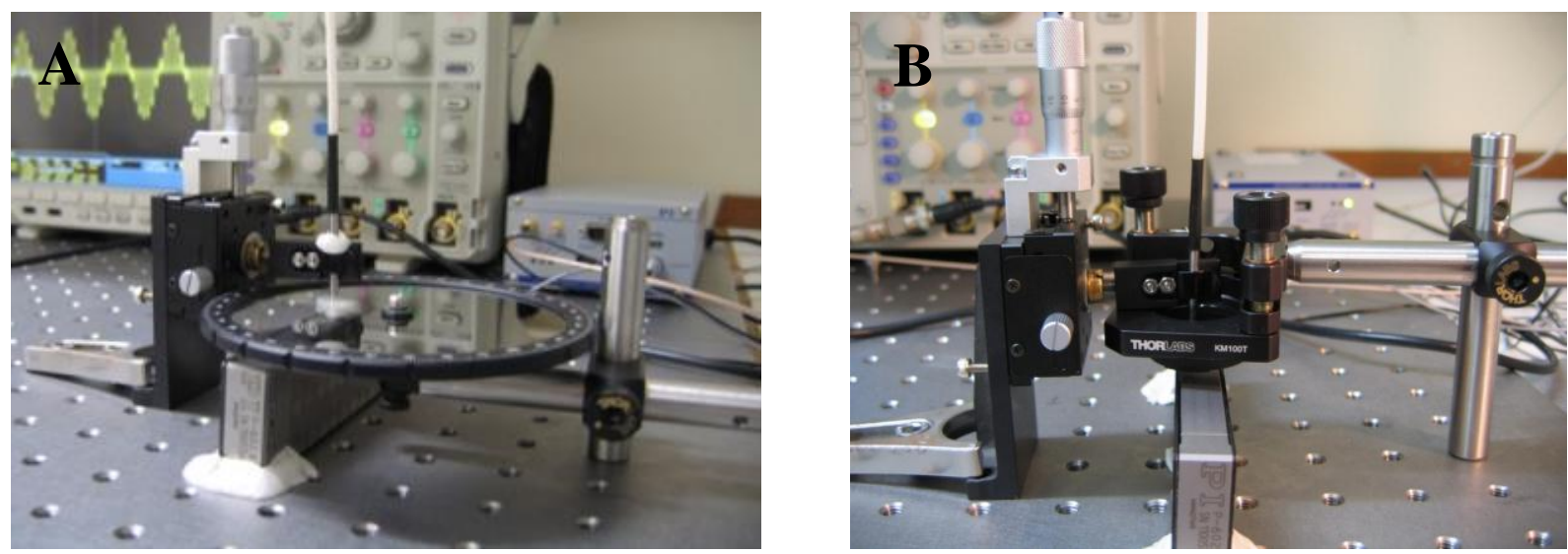

Figure 2. Experimental setups of measure for calibration of LDF devices.

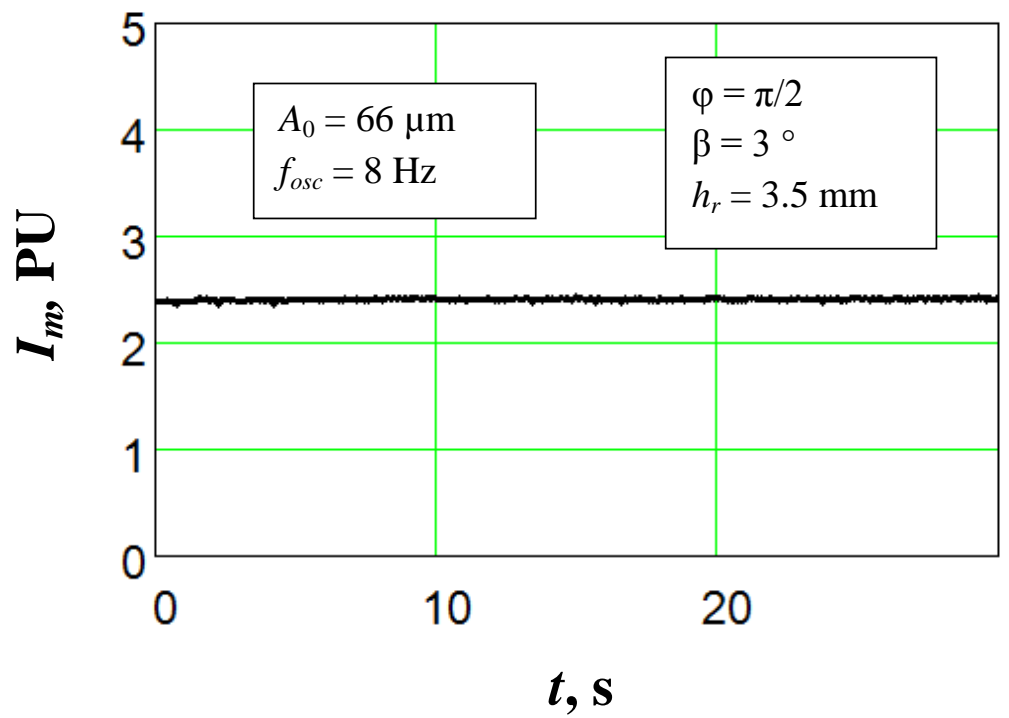

Figure 3. Example of reproducible constant level of perfusion using the proposed measure.

It should be emphasized that the proposed experimental setup is virtually insensitive to environmental vibration artefacts (Fig. 3), while use of colloidal phantoms for calibration requires an optical table with vibration damping (elimination of footsteps, closing doors, etc.).

Fig. 4 shows the graphs of the reproducible perfusion level on the amplitude (Fig. 4a) and frequency (Fig. 4b) of oscillation light scattering surface (on the experimental points with pending standard deviations added theoretical curve). Experimental and theoretical research has shown a linear portion for LDF-signal dependence on the amplitude of the oscillating layer $(0-70 \mu \mathrm{m})$ and also a linear portion $(0-10 \mathrm{~Hz})$ for LDF-signal from the oscillating frequency of the light scattering surface of the moving diffuse reflector. It should be emphasized that the experimental measure setups used in both cases (the range of changes in the amplitude and the frequency of oscillations) are consistent with the range of RBC 
velocity $(0-4.5 \mathrm{~mm} / \mathrm{s})$. A linear dependence of this function of the reproducible perfusion level allows the application of the proposed principle for calibration and adjustment of LDF devices.

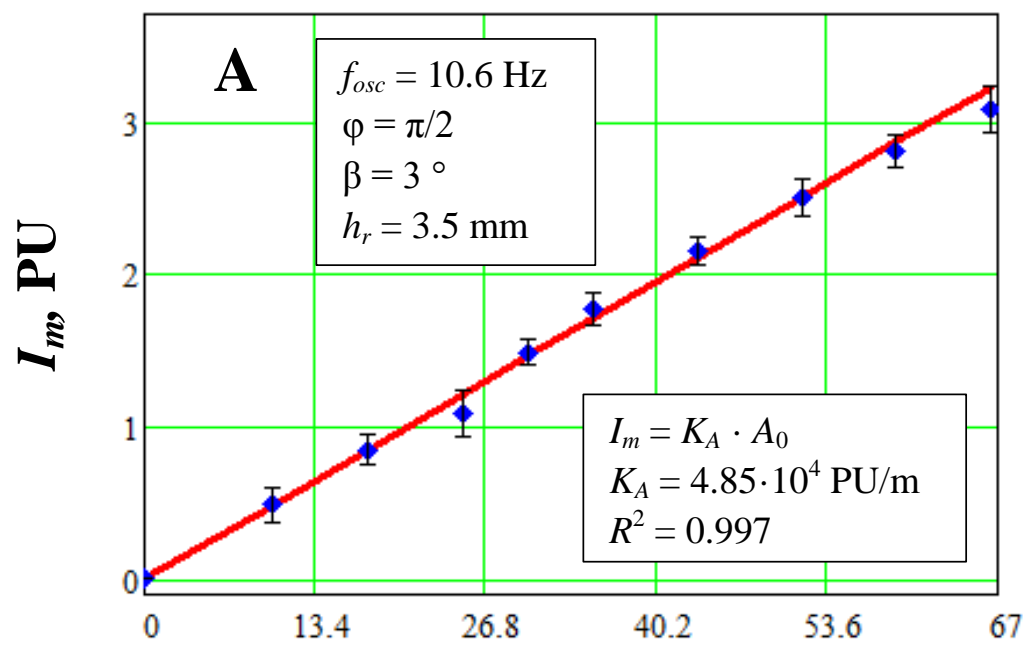

\section{$A_{0}, \mu \mathrm{m}$}

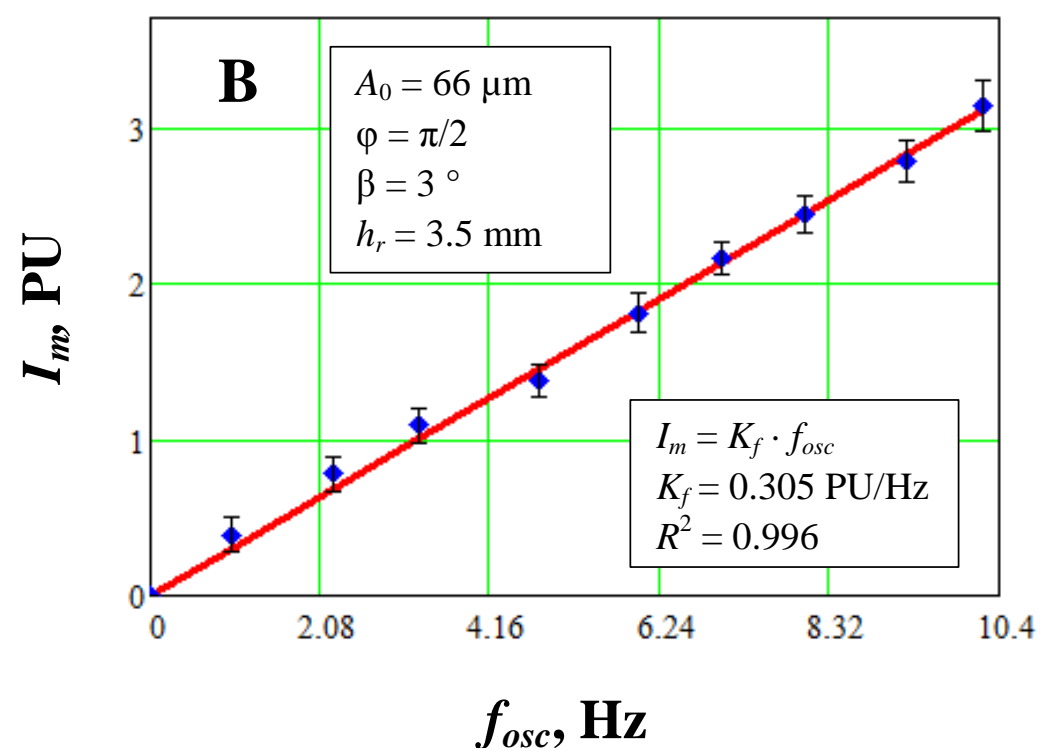

Figure 4. Experimental and theoretical dependencies reproducible levels of perfusion from the amplitude (a) and frequencies (b) oscillation of the light scattering surface of moving diffuse reflector.

For experimental verification of the type of function of reproducible perfusion level from the design parameters $\left(h_{r}-\right.$ distance from the plane of the fiber probe to the light scattering surface), a series of experiments was performed and the graph shown in Fig. 5 was constructed. The obtained graph dependence shows an inverse relationship of the reproducible perfusion levels on the distance from the light scattering surface. This result can be explained by the reduction of the solid angle subtended by the intersection area of the probe and reception areas of the center point of the core of the receiving fiber. The results suggest the possibility of applying the considered structure as a measure for calibration of LDF devices. 


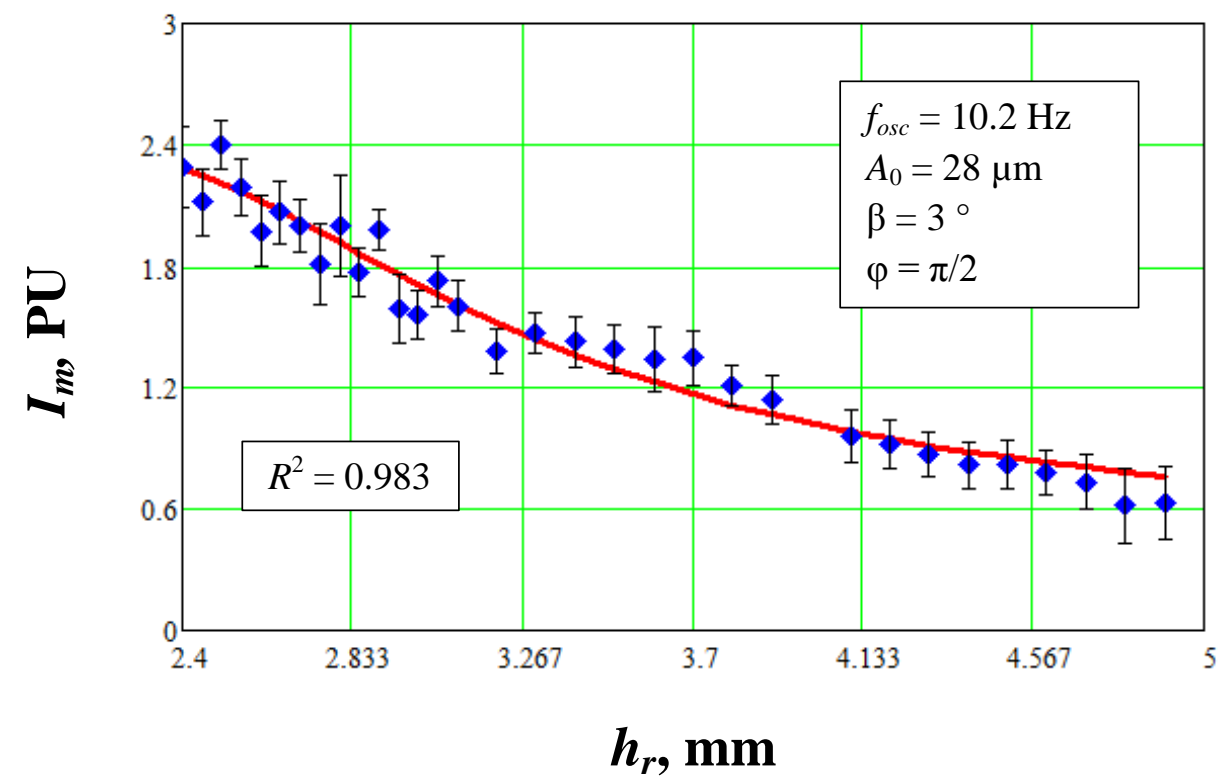

Figure 5. Experimental and theoretical dependencies reproducible levels of perfusion from the distance from the plane of the fiber probe to the light scattering surface of moving diffuse reflector.

\section{CONCLUSION}

Analysis of the experimental data shows the convenience and ease of operation of the proposed technique for the calibration of LDF devices, operational manageability of its modes and operability evaluation in devices systems of analysis of frequency rhythms of blood flow, which allows comparing them with each other. Proposed novel measure may be used to determine both static and dynamic errors of LDF devices. This allows evaluation of the suitability of LDF devices during production and specific diagnostic use in medical institutions. Developed algorithms for checking the metrological characteristics of LDF devices offer the possibility to improve the quality and reliability of the resulting diagnostic information.

\section{ACKNOWLEDGMENTS}

This work was supported by the European Community's Seventh Framework Programme (FP7-People-2009-IAPP) under Grant Agreement no. 251531 MEDILASE and partially by the grant of State University ESPC no. VK-3-2013.

\section{REFERENCES}

[1] Rogatkin, D. A., Dunaev, A. V. and Lapaeva, L. G., "Metrological Support of Methods and Devices for Noninvasive Medical Spectrophotometry," Biomedical Engineering, 44(2), 66-70 (2010).

[2] Obeid, A. N., Barnett, N. J., Dougherty, G. and Ward, G., "A critical-review of laser doppler flowmetry," Journal of Medical Engineering \& Technology, 14(5), 178-181 (1990).

[3] Leahy, M. J. and Nilsson, G. E., "Laser Doppler flowmetry for assessment of tissue microcirculation: 30 years to clinical acceptance," Proc. SPIE. 7563, 75630E (2010).

[4] Tuchin, V. V., [Handbook of optical biomedical diagnostics], SPIE Press, Bellingham, Washington(2002).

[5] Dunaev, A. V., Zherebtsov, E. A., Rogatkin, D. A., Stewart, N. A., Sokolovski, S. G. and Rafailov, E. U., "Substantiation of medical and technical requirements for noninvasive spectrophotometric diagnostic devices," Journal of Biomedical Optics, 18(10), 107009 (2013). 
[6] Binzoni, T., Leung, T. S., Seghier, M. L. and Delpy, D. T., "Translational and Brownian motion in laser-Doppler flowmetry of large tissue volumes," Physics in Medicine and Biology, 49(24), 5445-5458 (2004).

[7] Fredriksson, I., Larsson, M., Salomonsson, F. and Strömberg, T., "Improved calibration procedure for laser Doppler perfusion monitors," Proc. SPIE. 7906, 790602 (2011).

[8] Liebert, A., Leahy, M. and Maniewski, R., "A calibration standard for laser-Doppler perfusion measurements," Review of Scientific Instruments, 66, 5169 (1995).

[9] Bracic, M. and Stefanovska, A., "Wavelet-based analysis of human blood-flow dynamics," Bulletin of Mathematical Biology, 60(5), 919-935 (1998).

[10] Tankanag, A. and Chemeris, N., "Application of the adaptive wavelet transform for analysis of blood flow oscillations in the human skin," Physics in Medicine and Biology, 53(21), 5967-5976 (2008).

[11] Liebert, A., Leahy, M. and Maniewski, R., "Multichannel laser-Doppler probe for blood perfusion measurements with depth discrimination," Medical \& Biological Engineering \& Computing, 36(6), 740-747 (1998).

[12] Soelkner, G., Mitic, G. and Lohwasser, R., "Monte Carlo simulations and laser Doppler flow measurements with high penetration depth in biological tissuelike head phantoms," Applied Optics, 36(22), 5647-5654 (1997).

[13] Larsson, M., Steenbergen, W. and Stromberg, T., "Influence of optical properties and fiber separation on laser Doppler flowmetry," Journal of Biomedical Optics, 7(2), 236-243 (2002).

[14] Steenbergen, W. and de Mul, F. F. M., "Application of a novel laser Doppler tester including a sustainable tissue phantom," Proc. SPIE. 3252, 14-25 (1998).

[15] Rogatkin, D. A., Sokolovski, S. G., Fedorova, K. A., Sidorov, V. V., Stewart, N. A. and Rafailov, E. U., "Basic principles of design and functioning of multifunctional laser diagnostic system for non-invasive medical spectrophotometry," Proc. SPIE. 7890, 78901H1 (2011).

[16] Dunaev, A. V., Sidorov, V. V., Stewart, N. A., Sokolovski, S. G. and Rafailov, E. U., "Laser reflectance oximetry and Doppler flowmetry in assessment of complex physiological parameters of cutaneous blood microcirculation," Proc. SPIE. 8572, 857205 (2013). 